\title{
Avaliação da qualidade de vida em pacientes laringectomizados: uma revisão sistemática
}

\author{
Quality of life assessment in laryngectomized \\ patients: a systematic review
}

Arlinda B. Moreno 1

Claudia S. Lopes 1

\footnotetext{
1 Departamento de Epidemiologia, Instituto de Medicina Social, Universidade do Estado do Rio de Janeiro. Rua São Francisco Xavier 524, 7o andar, Bloco D, Rio de Janeiro, $R J$ 20550-900, Brasil.
}

\begin{abstract}
Laryngectomy is the main sequela in patients with cancer of the larynx. The authors conducted a systematic review to evaluate the relationship between quality of life and laryngectomy. Ninety-six articles published in scientific journals were identified, and 35 were selected whose main focus was laryngectomy and quality of life in laryngectomized patients. Each article was evaluated by way of systematic review. Evaluation of the qualitative attributes of articles used the Qualitative Assessment Questionnaire (QAQ), a validated and tested instrument. Most articles lacked consistency and methodological rigor in measuring quality of life among laryngectomized patients. The relationship between quality of life and laryngectomy also fell far short of the multidimensional approach of the quality of life construct as suggested by the WHO/QOL (World Health Organization/Quality of Life) group.
\end{abstract}

Key words Laryngectomy; Quality of Life; Evaluation

Resumo Laringectomia é a principal seqüela em pacientes com câncer de laringe. Neste estudo, as autoras conduziram uma revisão sistemática para avaliar a relação entre qualidade de vida e laringectomia. De 96 artigos publicados em periódicos científicos, previamente identificados, foram selecionados 35 artigos sobre laringectomia e qualidade de vida em pacientes laringectomizados. Todos os artigos selecionados foram submetidos à abordagem metodológica de uma revisão sistemática. Para a avaliação dos atributos qualitativos dos artigos foi utilizado o Questionário de Avaliação Qualitativa (QAQ), um instrumento testado e validado. Os resultados encontrados mostraram que a maior parte dos artigos selecionados apresentava inconsistências e falta de rigor metodológico na mensuração do constructo qualidade de vida entre pacientes laringectomizados. Além disso, verificou-se que a relação entre qualidade de vida e laringectomia, na forma apresentada nos artigos selecionados, ainda se encontra distante da abordagem multidimensional do constructo qualidade de vida, conforme preconizado pelo WHOQOL (World Health Organization - Quality of Life Group).

Palavras-chave Laringectomia; Qualidade de Vida; Avaliação 


\section{Introdução}

O câncer de laringe, como uma neoplasia comum de cabeça e pescoço, representando cerca de $25 \%$ dos tumores malignos desta região, pode ser, também, considerado doença rara, já que a sua incidência equivale a aproximadamente $1 \%$ de todas as lesões malignas do organismo humano (DeVita et al., 1997; Noronha \& Dias, 1997).

No Brasil, em 1990, a estimativa da Organização Mundial de Saúde (OMS), para a incidência deste tipo de tumor, era de 7,79 , para homens e 1,06, para mulheres, por 100.000 habitantes (taxas já padronizadas por idade). Além disso, a taxa de mortalidade estimada para câncer de laringe era de 4,76 para homens e de 0,65 para mulheres, por 100.000 habitantes. Em termos mundiais, as taxas de incidência e de mortalidade alcançaram os seguintes números: (a) incidência igual a 5,69 para homens e 0,72 para mulheres, por 100.000 habitantes; $\mathrm{e}$ (b) mortalidade igual a 3,12 e 0,37 para homens e mulheres, respectivamente, para 100.000 habitantes. Tais dados indicam, portanto, que as taxas brasileiras apresentam-se mais elevadas do que a média em termos globais (WHO/ IARC, 1999).

A faixa etária mais atingida por este tipo de tumor, tanto para homens como para mulheres, é aquela superior a 55 anos (Kligerman et al., 1992).

Na etiologia do câncer de laringe, tem-se que o fumo e a ingesta pesada de álcool representam os fatores de risco mais comuns (Cattaruzza et al., 1996; López-Abente et al., 1992; Zatonski et al., 1991). Todavia, fatores relacionados à dieta e à exposição à serragem, ao pó de madeira e ao asbesto também têm sido relatados na literatura (Muscat \& Wynder, 1992; Wortley et al., 1992; Zheng et al., 1992).

Embora as taxas de cura de várias malignidades da laringe não tenham mudado consideravelmente durante os anos recentes, as investigações sobre métodos de conservação da laringe, quando se dá a ameaça de laringectomia, bem como de diversas estratégias de radioterapia e combinações de quimio-radioterapia, têm sido incessantemente consideradas no tratamento do paciente com câncer de laringe (DeVita et al., 1997). Assim, principalmente quando referido a tumores avançados, o tratamento cirúrgico indicado implica mutilação por conta da extração da laringe e, conseqüentemente, das cordas vocais. O paciente laringectomizado é, sem dúvida, alguém que sofreu uma mutilação e que, por conta da cirurgia laríngea, deve passar a viver sob o estigma do traqueostoma e sob grande impacto em sua capacidade de comunicar-se por meio da voz. Desta forma, cerca de $50 \%$ desta população se retira do convívio social, perdendo, também, seus empregos. Ressalta-se, ainda, que não se trata tão somente de cotejar qualidade de vida e/ou morte nestes doentes (no que se refere a questões funcionais), uma vez que a ameaça ao órgão vocal do paciente está associada a profundas implicações psicológicas (Gunzburger, 1997; Kligerman et al., 1992).

Note-se, também, que um considerável número de trabalhos científicos tem sido publicado acerca dos efeitos da laringectomia na sobrevida do paciente, o que suscita a necessidade de se rever criticamente a bibliografia existente sobre o tema (DeVita et al., 1997; Mathieson et al., 1990). A qualidade de vida do paciente que sofre a retirada da laringe e, conseqüentemente, de suas cordas vocais, tem, em muitos destes trabalhos, recebido especial ênfase (Kligerman et al., 1992; Mohide et al., 1992).

Assim sendo, em parceria com o desenvolvimento técnico-científico que a medicina tem galgado, questões relativas à qualidade de vida dos portadores de moléstias graves, entre elas o câncer, têm sido efetivamente consideradas. Com isto, uma diversidade de instrumentos para mensuração de tais questões tem sido montada, estruturada, desenvolvida, consolidada e aplicada a boa parte dessa população (Bowling, 1995a).

Esta diversidade parece refletir uma falta de homogeneidade no conceito. Nesse sentido, em 1993, o World Health Organization - Quality of Life Group (WHOQOL), definiu qualidade de vida como: "qualidade de vida é definida como uma percepção individual da posição do indivíduo na vida, no contexto de sua cultura e sistema de valores nos quais ele está inserido e em relação aos seus objetivos, expectativas, padrões e preocupações. É um conceito de alcance abrangente afetado de forma complexa pela saúde física, estado psicológico, nível de independência, relações sociais e relações com as características do meio ambiente do indivíduo" (WHO, 1993:1).

Este estudo tem como objetivo realizar uma revisão sistemática de artigos acerca de laringectomia e qualidade de vida, objetivando avaliar as relações existentes entre estes dois constructos, quando referidos a pacientes laringectomizados. 


\section{Metodologia}

\section{Revisão sistemática}

Com base no trabalho de Oliveira (1998), foi adaptada uma estratégia metodológica básica, integrando regras sugeridas em duas linhas de pesquisa: meta-análise de ensaios clínicos (Anello \& Fleiss, 1995; Jenicek, 1989; Sacks et al., 1987) e revisões sistemáticas da literatura (Oxman \& Guyatt, 1988, 1993), conforme a seguir:

- Especificação do objeto

- Objetivos do estudo

- Especificação da estratégia de busca bibliográfica

- Definição dos critérios de inclusão/exclusão dos artigos

- Questionário de avaliação qualitativa

- Resultados:

a) Da busca bibliográfica

b) Da avaliação da validade do questionário

c) Da aplicação do questionário

d) Quantificação dos principais achados

e) Classificação qualitativa

- Discussão e conclusões apoiadas nos resultados

- $\quad$ Sugestões para futuras pesquisas

\section{Objetivos e levantamento bibliográfico}

O objeto de estudo são artigos científicos apresentando resultados que correlacionem laringectomia e qualidade de vida, sendo o objetivo principal avaliar as relações entre qualidade de vida e laringectomia, por meio da realização de uma revisão sistemática. A identificação de artigos sobre laringectomia e qualidade de vida em pacientes laringectomizados se deu: (a) por meio de execução de busca bibliográfica em bancos de dados informatizados, mais precisamente: MEDLINE, Current Contents, LILACS e Cochrane Lybrary; e (b) por meio de execução de busca manual em listas de referência dos artigos identificados e selecionados; uso de palavras-chave, em periódicos ligados ao tema, disponíveis em bibliotecas do Rio de Janeiro; contato com especialistas ou bancos de artigos não publicados que tenham relevância para o contexto investigado.

As palavras-chave utilizadas na identificação dos artigos foram: "quality of life AND laryngectomy" e seus equivalentes em português e em espanhol ("qualidade de vida AND laringectomia" e "calidad de vida AND laringectomía").

Quanto à definição dos Critérios de Inclusão/Exclusão dos artigos, temos que:

- Critérios de Inclusão: (a) artigos originais de pesquisa (excluindo-se editoriais e relatos de casos) sobre laringectomia e qualidade de vida em pacientes laringectomizados, em um intervalo de tempo compreendido entre 1966 (ano inicial dos registros do MEDLINE) e agosto/1998 e que tenham sido publicados em português, espanhol, francês, inglês e italiano; e (b) estudos apresentados em Congressos e Seminários, estudos governamentais, dissertações e teses, que estejam, também, inseridos no intervalo de tempo acima citado ("literatura fugitiva"). - Critério de Exclusão: artigos que sejam relativos à descrição de técnicas cirúrgicas, bem como artigos de revisão de literatura e/ou que não circunscrevam uma população de laringectomizados.

\section{Questionário de Avaliação Qualitativa (QAQ)}

Para a avaliação dos atributos qualitativos dos artigos, foi utilizado o Questionário de Avaliação Qualitativa (QAQ). Esse instrumento, testado e validado, foi estruturado em cinco seções com a intenção de mensurar dimensões relativas: (a) à identificação do artigo (tipo de avaliação sobre qualidade de vida que o estudo pretende demonstrar); (b) às características metodológicas do estudo (relação do tipo de desenho do estudo com as dimensões sobre qualidade de vida exploradas); (c) às características gerais da amostra (tais como idade, nível de escolaridade, ocupação principal, entre outras); (d) às características específicas da população com tumor de laringe (tipo de tumor de laringe que promoveu a laringectomia, seu estádio e localização, terapêuticas ministradas, estratégias de recuperação/preservação de voz); e (e) à qualidade de vida (utilização ou não de instrumentos para a mensuração da qualidade de vida)

Para a composição do QAQ, utilizamo-nos da consideração que Oxman \& Guyatt (1991) fazem acerca do desenvolvimento de critérios para avaliação de qualidade da literatura, que eles julgam terem forma análoga ao desenvolvimento de questionários utilizados para mensuração de qualidade de vida, o que neste trabalho se apresenta como bastante apropriado. Além disso, segundo Streiner \& Norman (1989), conceituação, geração e seleção dos itens, formação de escalas e validação do instrumento por meio de testes de confiabilidade e validade são etapas imprescindíveis à construção de instrumentos para avaliação qualitativa, sendo esta idéia compartilhada por Oxman \& Guyatt (1991).

Para avaliar a validade de face/conteúdo do QAQ, este foi submetido a seis especialistas (professores com Mestrado ou Doutorado nas 
áreas de Epidemiologia e Cancerologia), que responderam a um questionário com treze itens, desenvolvido e validado por Oxman \& Guyatt (1991), cuja pontuação varia de zero a sete (assim, caso o instrumento alcance, na apreciação de um especialista, seis em sete pontos, em um dado item, este terá como "nota final" [(6/7)*100] ou $85,71 \%$ de pontuação). Este questionário de validação foi estruturado com o intuito de verificar se o QAQ era um instrumento que: (a) poderia ser aplicado a uma extensa variedade de artigos sobre o tema; (b) poderia ser utilizado por pesquisadores na área de epidemiologia; (c) possuía itens claros e precisos; (d) tinha instruções adequadas para aplicação; (e) não necessitava de informações que não estivessem contidas nos artigos selecionados; (f) prescindia de decisões subjetivas para escolha de respostas adequadas; (g) tinha forma e conteúdo que não gerassem vieses nas respostas; (h) aferia um único domínio; (i) prescindia de itens redundantes e desnecessários; (j) não excluía itens importantes sobre a qualidade dos estudos sobre o tema; (k) tinha pontuação adequada para os itens; (l) tinha opções de respostas suficientes para os itens; (m) tinha poder discriminativo para discernir artigos de boa e má qualidade científica.

Assim, o instrumento obteve como resultado da aplicação do questionário de validação uma avaliação percentual, de acordo com a pontuação acima referida, que variou de $75,8 \%$ a $93,9 \%$, com média global igual a $79,8 \%$, o que foi considerado satisfatório. Todavia, três dos 13 itens (aplicabilidade ampla, informações disponíveis e domínio único a ser mensurado) obtiveram média inferior a cinco em sete pontos (ou 71,43\%), índice considerado por Oxman \& Guyatt (1991) como nota mínima para cada um dos 13 critérios examinados.

A confiabilidade interobservador foi testada em oito artigos mediante o Coeficiente de Correlação Intraclasse (ICC), para comparar as respostas da autora (A. B. M.) e de um outro observador com Mestrado em Epidemiologia. Com isso, pretendeu-se avaliar o quanto as respostas extraídas pelo observador 1 (autora A. B. M.) estavam em consonância com aquelas emitidas pelo observador 2 (epidemiologista), quando ambos utilizavam o QAQ, ou seja, avaliar o quanto o instrumento permanece estável quando utilizado por mais de um extrator. Os dois observadores aplicaram o QAQ de forma independente e foi avaliada a concordância entre as pontuações (medidas contínuas) atribuídas aos artigos.

As pontuações atribuídas observavam os seguintes critérios: (a) Reconhecimento de possí- veis vieses de seleção/aferição; (b) Utilização de estratégias para minimização de vieses; (c) Reconhecimento de variáveis de confusão; (d) Utilização de estratégias para controle de variáveis de confusão; (e) Localização do tumor que suscitou a laringectomia; (f) Agregação dos grupos de estudo por estádio do tumor; (g) Terapêutica ministrada com o objetivo da preservação da voz; (h) Avaliação da Qualidade de Vida por intermédio da aplicação de instrumento específico; (i) Avaliação de validade do instrumento aplicado para mensuração da Qualidade de Vida, quando este não foi padronizado previamente; (j) Avaliação da confiabilidade do instrumento aplicado para mensuração da Qualidade de Vida, quando este não foi padronizado previamente; (k) Respostas acerca da avaliação da Qualidade de Vida emitidas pelo próprio paciente; (l) Sugestões para melhorias futuras.

Dessa forma, cada um dos critérios acima que obteve como resposta um SIM forneceu um ponto ao artigo em questão; as respostas NÃO, N.I. (Não Informado) e N.A. (Não se Aplica) não forneceram pontuação ao artigo, podendo, portanto, cada artigo alcançar um máximo de 12 pontos, por observador.

A despeito de o observador 2 ter atribuído pontuação levemente maior aos artigos do que o observador 1, quando da estimação do ICC, foi alcançada uma confiabilidade de 0,9045 (IC 95\%: 0,6118-0,9808). Um detalhamento sobre o QAQ pode ser verificado no trabalho de Moreno (1999).

\section{Análise de dados}

Para a montagem do banco de dados reunindo todas as respostas lançadas no QAQ e para a análise de freqüência das variáveis, foi utilizado o Epi Info 6, Versão 6.02 (WHO, 1994). A análise do ICC (cálculo do intervalo de confiança de 95\%), foi realizada no SPSS for Windows, Versão 8.0 (SPSS Incorporation, 1997). Além disso, para a geração da análise de variância a 2 fatores (para cálculo do coeficiente de correlação intraclasse) e composição de gráficos e tabelas, o Excel para Windows 95, Versão 7.0, foi utilizado.

\section{Resultados}

\section{Busca bibliográfica}

De acordo com os critérios de inclusão/exclusão, 71 artigos foram localizados. A leitura integral dos mesmos resultou na seleção final de 35 
artigos. Nesta fase, os principais motivos para exclusão de cerca de $51 \%$ dos artigos foram: artigos de revisão, estudo de caso e descrição de técnicas cirúrgicas. A busca manual não trouxe novos artigos à amostra previamente selecionada.

\section{Identificação do artigo}

Cerca de $51 \%$ dos artigos foram promovidos por instituições hospitalares, $40 \%$ por universidades e os $9 \%$ restantes por centros de pesquisas e outras entidades. Dos 35 estudos analisados, $85,7 \%$ (trinta artigos) eram realizados por apenas uma instituição (ou seja, sem a colaboração de outra instituição correlata - hospitalar, ensino etc.).

Quanto ao país de publicação dos estudos analisados, temos os Estados Unidos com 48,6\% (17 artigos), seguido dos Países Baixos, com 14,3\% (cinco artigos) e da Itália com 8,9\% (três artigos). Canadá e Inglaterra contribuíram, cada um deles, com dois artigos e Brasil, China, Índia, Nova Zelândia, Noruega e Reino Unido, com um artigo cada.

Quanto ao ano de publicação dos artigos, tem-se que o primeiro artigo incluído no estudo foi publicado em 1977 e que a maioria deles $(71,4 \%)$ foi publicada a partir de 1990.

Os periódicos em que foram publicados os artigos selecionados tinham como especialidade, em 62,9\% dos casos, a otorrinolaringologia, seguidos da cancerologia em 22,9\% dos artigos. Medicina interna $(8,6 \%)$, cirurgia $(2,9 \%)$ e psiquiatria $(2,9 \%)$ compunham o restante da distribuição.

\section{Características metodológicas do estudo}

Dos 35 artigos analisados, 27 (77,1\%) dos estudos tinham desenho transversal, sendo a grande maioria inquéritos. Eram de coorte cinco estudos, dois eram ensaios clínicos e apenas um estudo era caso-controle.

Proporções (68,6\% dos artigos) e médias (31,4\% dos artigos) foram as medidas de freqüência mais utilizadas, além de coeficientes de correlação e taxas de sobrevida.

As análises estatísticas mais comuns foram os testes de médias (37,1\% dos artigos) e de proporções $(11,4 \%$ dos artigos), além de análises de variância (ANOVA e MANOVA) e de sobrevida, regressão linear e logística.

Dos 35 artigos selecionados, temos que em $29(82,9 \%)$ deles a avaliação de vieses não foi realizada. Assim, apenas seis dos 35 artigos $(17,1 \%)$ consideraram a possibilidade de vieses em seus achados, e destes, três consideraram vieses de aferição e três de seleção. Note-se que apenas dois, de todos os artigos, declararam o uso de estratégias para minimização desses vieses.

Quanto às variáveis de confusão, dos 23 artigos em que a avaliação de tal característica era pertinente, apenas quatro $(17,4 \%)$ consideraram que seus achados poderiam estar distorcidos, mas apenas dois se utilizaram de estratégias para controle de tais variáveis.

\section{Características gerais da amostra}

Foram analisados aspectos relativos à amostra de indivíduos selecionados para compor o estudo conforme dados apresentados na Tabela 1 .

Um total de 3.738 indivíduos foi abarcado pelos 35 artigos selecionados. A Tabela 1 mostra, entre outras características, que em 27 artigos a média de idade era superior a 55 anos e que, em média, $85 \%$ da população era masculina. Além disso, temos que o total de não-respondentes é igual a 367 indivíduos, tendo esta característica sido mencionada em 20 dos 35 artigos.

Além disso, a proporção de indivíduos do sexo masculino e feminino, ao longo do período, apresentou-se de modo bastante regular, tendo prevalecido a maioria masculina (cerca de $85 \%$, em média, das amostras), no que se refere à população afetada pelo câncer de laringe.

Os países nos quais os estudos foram realizados (o que não coincide com os países que publicaram os estudos) estão assim distribuídos: (a) Estados Unidos - 37,1\% (13 estudos); (b) Países Baixos - 14,3\% (cinco estudos); (c) Canadá e Itália - três estudos cada; (d) Inglaterra - dois estudos; e (e) Austrália, Bélgica, Brasil, China, Índia, Nova Zelândia, Noruega, Reino Unido e Suécia - cada um deles com um estudo.

A distribuição da base populacional mostrou que a maioria dos estudos trabalhou com amostras de base hospitalar $(57,1 \%$ geral e $25,7 \%$ universitária), sendo apenas $11,4 \%$ de base comunitária.

\section{Características específicas do tumor} de laringe (localização, estádio, estratégia terapêutica utilizada, estratégia de recuperação da voz)

Quanto a estas características, temos que: a) Nove estudos $(25,7 \%)$ informaram a localização do tumor de laringe que promoveu a laringectomia (ou a estratégia terapêutica utilizada); 
Tabela 1

Características gerais da amostra.

\begin{tabular}{|c|c|c|}
\hline Evento & Número de artigos & $\%$ \\
\hline Seleção da amostra por censo $(n=3.019)$ & 25 & 71,4 \\
\hline Seleção por amostragem $(n=719)$ & 10 & 28,6 \\
\hline Número de não respondentes $(n=367)$ & 20 & 57,1 \\
\hline Média de idade superior a 55 anos* & 27 & 77,1 \\
\hline Nível de escolaridade básico & 5 & 14,3 \\
\hline Nível de escolaridade médio & 1 & 2,9 \\
\hline Ocupação principal (aposentados - 1 artigo; bombeiros e executivos - 1 artigo) & 2 & 5,7 \\
\hline Raça da maioria dos indivíduos (análises focadas sobre a raça branca) & 4 & 11,4 \\
\hline Distribuição por sexo (média de população masculina = 85\%) & 31 & 88,6 \\
\hline $\begin{array}{l}\text { Período de realização/duração do estudo (média de duração = } 8 \text { meses - amplitude } \\
\text { igual a } 2 \text { a } 35 \text { meses) }\end{array}$ & 9 & 25,7 \\
\hline
\end{tabular}

* Seis artigos $(17,1 \%)$ não informaram a média de idade dos indivíduos.

b) Onze estudos $(31,4 \%)$ indicaram o estádio dos tumores e consideraram estes dados em suas análises de resultados;

c) Trinta e quatro estudos $(97,1 \%)$ se utilizaram de um ou mais esquemas terapêuticos na população estudada. O único artigo que não o fez tratava da avaliação da Qualidade de Vida em indivíduos sadios (componentes do corpo de bombeiros e executivos).

Dentre as estratégias terapêuticas escolhidas para o grupo principal (ou único) do estudo e para o segundo grupo, temos que cirurgia (associada ou não a quimioterapia e/ou a radioterapia) foi o tratamento de escolha seguido pela radioterapia;

d) Vinte e oito artigos $(82,4 \%)$ citaram a estratégia terapêutica "laringectomia total" na população estudada;

e) No que se refere ao fato de a cirurgia escolhida como estratégia terapêutica tentar privilegiar a preservação da voz nos indivíduos, temos que seis destes artigos citaram ser a escolha terapêutica devida a este fato. Note-se, todavia, que em 15 dos estudos este tipo de questionamento teve resposta igual a N.A., uma vez que não cabia este tipo de observação no propósito e objetivo de tais estudos. Dos seis artigos citados como tendo este tipo de preocupação, quatro deles puderam estender esta estratégia terapêutica a $100 \%$ da população, um deles a $53 \%$ e outro a apenas $21 \%$ dos indivíduos;

f) Dezenove artigos citaram a estratégia de recuperação de voz utilizada após a cirurgia, de forma não excludente: (a) treinamento fonoaudioterápico - 15 artigos; (b) laringe eletrônica - 11 artigos; (c) uso de prótese - nove artigos; e (d) outras estratégias (reconstrução, escrita etc.) - cinco artigos.

\section{Qualidade de vida}

Dos 35 artigos estudados, 45,7\% (16 artigos) se utilizaram de instrumentos para a avaliação da Qualidade de Vida na população estudada, sendo o uso de instrumentos padronizados relatado em nove artigos e o de instrumentos não padronizados mencionado em 14 artigos. Deve-se considerar, assim, que houve estudos que se utilizaram de mais de um instrumento para a sua pesquisa (seja padronizado ou não). A Tabela 2 apresenta uma distribuição do uso de instrumentos padronizados tendo sido o European Organization for Research and Treatment of Cancer Core - Quality Life - Questionnaire 30 (EORTC-QLQ-C-30) o de mais ampla utilização $(8,5 \%$ dos artigos). Todavia, existe uma ampla variabilidade de instrumentos que estão sendo utilizados pelos estudos (16 outros instrumentos são listados na Tabela 2).

A distribuição ao longo do tempo, do uso de instrumentos para avaliação de qualidade de vida (Figura 1), mostrou que a utilização de instrumentos padronizados tem início no ano de 1994 e que, em contrapartida, desde 1986, instrumentos para avaliação de qualidade de vida têm sido utilizados.

Quanto às dimensões privilegiadas pelos estudos que se utilizaram de algum instrumento para avaliação da Qualidade de Vida, temos que Relações Sociais (em 100\% dos artigos) e Saúde Física (em 15 dos 16 artigos) são as dimensões que mais suscitaram investigações pelos autores. Além disso, Estado Psicológico e 
Instrumentos padronizados utilizados pelos artigos.

\begin{tabular}{lc}
\hline Título do instrumento padronizado & Freqüência \\
\hline Atkinson Life Happiness Rating & 1 \\
CES-D (Center for Epidemiologic Studies Depression) & 1 \\
EORTC-H\&N-37 (European Organization for Research and Treatment of Cancer - Head and Neck - 37) & 1 \\
EORTC-QLQ-C-30 (European Organization for Research and Treatment of Cancer Core - Quality & 3 \\
Life- Questionnaire 30) & 1 \\
Escala Zung (depressão) & 1 \\
FACT-G Subscale (Functional Assessment of Cancer Therapy) & 1 \\
FACT-HN (Functional Assessment of Cancer Therapy) - Head and Neck & 1 \\
GHQ (General Health Questionnaire) & 1 \\
HAD (Hospital Anxiety and Depression Scale) & 1 \\
IIRS (IIIness Intrusiveness Ratings Scale) & 1 \\
Karnofsky Performance Status Rating Scale & 1 \\
Life Satisfaction & 1 \\
PAIS (Psychosocial Adjustment to IIlness Scale) & 1 \\
PSS-HN (Performance Status Scale for Head and Neck Cancer Patients) & 1 \\
SF-36 (Medical Outcomes Study 36-Item Short Form Health Survey) & 1 \\
STAI (State Trait Anxiety Inventory) & 1 \\
VHI (Voice Handcap Index) & 1 \\
\hline
\end{tabular}

Figura 1

Uso de instrumentos para avaliação de qualidade de vida

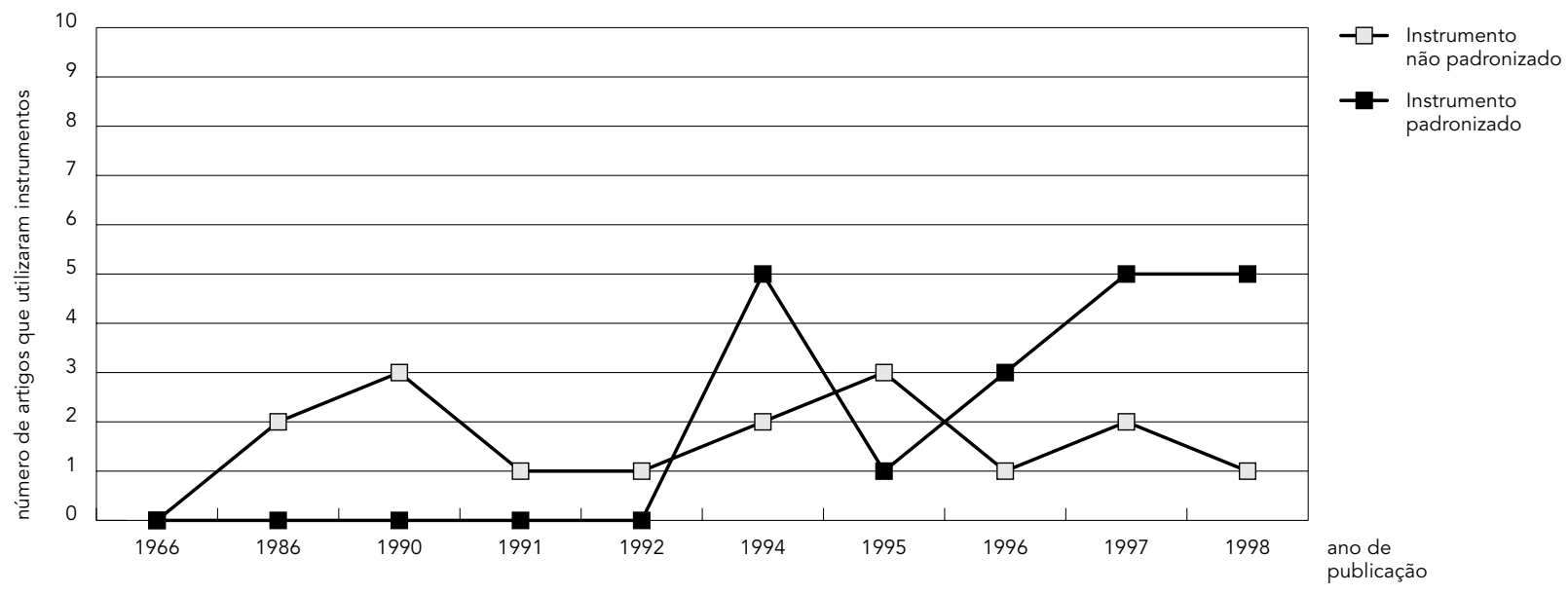


Relações com as Características do Meio Ambiente do Indivíduo estão citados em 13 e 11 estudos, respectivamente.

Quanto à avaliação de Validade e Confiabilidade dos instrumentos não padronizados e construídos especialmente para os estudos que estavam se desenvolvendo, apenas dois artigos mencionaram estratégias de avaliação da validade de seus instrumentos e três o fizeram no que se refere à confiabilidade dos mesmos.

Quando não se utilizaram instrumentos para a avaliação da Qualidade de Vida, foram avaliados os principais aspectos que, segundo os autores, estavam relacionados à qualidade de vida dos laringectomizados, tendo sido o aspecto Voz e/ ou Fala (avaliado em 12 dos 19 artigos) o mais privilegiado, seguido por Condições Físicas (avaliadas em 10 dos 19 artigos).

Quanto aos indivíduos que expressaram opiniões acerca dos dados sobre Qualidade de Vida que foram levantados nos artigos, temos como respondentes: (a) pacientes -27 artigos; (b) médicos - dez artigos; (c) equipe de saúde e familiares - quatro artigos; e (d) outros (voluntários sadios) - um artigo.

Dos 35 estudos analisados, apenas 14 (40\% dos artigos) sugeriram estratégias/condutas terapêuticas voltadas exclusivamente para a melhoria da qualidade de vida dos indivíduos estudados.

\section{Discussão}

Preliminarmente, o fato de terem sido selecionados por seus resumos 71 artigos, tendo sido incluídos na pesquisa apenas 49\% (35 estudos) deste total, pode estar nos remetendo a duas limitações no estudo: (a) algum problema na estratégia de busca dos artigos, podendo ter sido a mesma muito abrangente e, portanto, não muito eficaz para localizar estritamente a relação existente entre qualidade de vida e laringectomia; e (b) a uma provável tendência à corroboração de que boa parte dos artigos dizem que sua proposta é a avaliação de aspectos da qualidade de vida e, na realidade, tal objetivo não é alcançado - sobretudo porque muitos artigos tratam da descrição de técnicas e/ou terapêuticas específicas que serão responsáveis por um dado ganho de qualidade de vida para os pacientes laringectomizados, mas, na verdade, tal abordagem descrita pela equipe de saúde não é, segundo a definição de qualidade de vida que norteia este estudo (baseada na definição formulada pelo WHOQOL), uma avaliação deste constructo, seja porque a visão do paciente não foi privilegiada na descrição do evento estudado, seja porque apenas a dimensão saúde física é considerada quando este tipo de abordagem se dá.

A distribuição no tempo dos artigos analisados neste trabalho mostra que cerca de 4/5 do total de artigos analisados foram publicados entre 1986 e 1998. Tal fato tem a importância de trazer para este estudo a corroboração de que avaliar qualidade de vida é tarefa recente que tem tido um significativo crescimento ao longo do período (Batel-Copel et al., 1997; Boer et al., 1999; Camilleri-Brennan \& Steele, 1998).

No que se refere às características metodológicas dos estudos analisados, apenas cinco estudos eram estudos de coorte, o que traz para esta revisão, à semelhança do que se encontra em Boer et al. (1999) e Gotay \& Muraoka (1998), a possibilidade de mostrar que não existe um número significativo de estudos observacionais prospectivos que possam abarcar com maior especificidade a questão da qualidade de vida em pacientes laringectomizados, o que não se coaduna com o fato de que a sobrevida na maioria dos casos é considerada satisfatória (65\% de taxa de sobrevida global em cinco anos).

Em relação à possibilidade de vieses e a variáveis de confusão, apenas seis dos artigos selecionados consideraram a possibilidade de tais ocorrências em seus achados e somente dois artigos relataram as estratégias utilizadas para minimização de vieses e para controle de variáveis de confusão. Logo, tais fatos inviabilizaram uma discussão sobre fontes potenciais de vieses e de variáveis de confusão bem como dos métodos utilizados pelos estudos para minimizar tais efeitos.

A localização e estádio do tumor e o tipo de terapêutica escolhida para tratamento dos pacientes, foram assim informados: (a) nove artigos citaram a localização do tumor; e (b) 11 estudos indicaram o estádio dos tumores de laringe tratados. Tal fato surpreende negativamente, na medida em que avaliações sobre qualidade de vida em pacientes laringectomizados deveriam privilegiar informações sobre o tipo de tumor que suscitou a laringectomia, já que isto pode levar à melhor escolha de intervenções terapêuticas e, conseqüentemente, à minimização de seqüelas terapêuticas, com indubitável ganho na qualidade de vida que poderá ser proporcionada ao paciente. Além disso, avaliar qualidade de vida em pacientes cuja diversidade de seqüelas e mutilações seja muito ampla, não promove estudos que diferenciam com propriedade o que acontece com a população de laringectomizados. Não se po- 
de, por exemplo, avaliar qualidade de vida global em pacientes que puderam ter seu aparelho fonador funcionalmente preservado e laringectomizados totais, como se estes fizessem parte de um só grupo. Decerto que a qualidade de vida percebida por estes dois grupos de indivíduos é fortemente diferenciada, caso esta esteja sendo avaliada de acordo, obviamente, com as dimensões da qualidade de vida definida pelo WHOQOL. Este tipo de observação é também encontrada em Montazeri et al. (1998), em uma revisão de literatura sobre qualidade de vida em pacientes com câncer de pulmão, em que é feita uma diferenciação entre cânceres de pulmão de pequenas células ou não. Em última análise, pois, estamos falando do fato de a não-consideração da localização e estádio do tumor gerarem distorção nos estudos realizados - controlar por essas variáveis pode ser uma forma epidemiológica de controlar variáveis de confusão.

Além disso, note-se que $82,4 \%$ dos artigos citaram a estratégia terapêutica laringectomia total na população estudada e que, em média, $69,1 \%$ dos indivíduos arrolados nesses estudos se submeteram a este tipo de intervenção. Estes achados assinalam, minimamente, um certo retardo na descoberta do tumor de laringe e, conseqüentemente, perdas significativas na qualidade de vida, além de indicarem que técnicas cirúrgicas mais modernas, que podem ser utilizadas como escolha terapêutica para este tipo de população, ainda não estão largamente disponíveis. Considerando que a preservação da respiração normal também é elemento de grande peso nessa escolha, cabe registrar que, nos artigos restantes, esse fato também não foi privilegiado - não houve, portanto, intenção de preservação da respiração normal em detrimento da voz (fala).

Dos 35 artigos estudados, 45,7\% (16 artigos) se utilizaram de instrumentos para a avaliação da Qualidade de Vida na população estudada. Uma diversidade de 14 instrumentos padronizados foi utilizada nas pesquisas e pôde-se verificar que, conforme a recomendação de Bowling (1995a), escalas genéricas e escalas de domínios específicos foram utilizadas na mensuração de qualidade de vida em indivíduos laringectomizados. Além disso, os instrumentos padronizados escolhidos pelos estudos realizados mostraram-se adequados, à proporção que avaliaram bem-estar físico (sintomas tais como dor, fadiga, náusea, toxicidade à quimioterapia, atividade física e trabalho, recreação e atividades de autocuidado), bem-estar psicológico (imagem corporal, auto-estima, angústia emocional, raiva e depressão) e bem-estar social (efeitos nas atividades sociais, isolamento, suporte social da família e dos amigos e relações sexuais), sob a ótica do paciente (a maioria deles era preenchida pelo próprio paciente ou de acordo com sua percepção).

Cabe ainda ressaltar que foram desenvolvidos e/ou adaptados instrumentos para avaliação de qualidade de vida por 14 dos 16 artigos que se utilizaram de instrumentos para avaliação de tal constructo. Todavia (e em consonância com o achado de Gotay \& Muraoka, 1998), apenas dois artigos mencionaram estratégias para avaliação da validade de seus instrumentos e três o fizeram no referente à confiabilidade, o que denota uma preocupação quase nula em relação a dois aspectos metodológicos fundamentais para que seja fornecida magnitude epidemiológica aos estudos. Além disso, dos seis artigos que se utilizaram apenas de instrumentos não padronizados, nenhum deles citou estratégias de avaliação da validade de seus instrumentos e apenas um o fez em relação à confiabilidade.

Quanto às dimensões do constructo "Qualidade de Vida" que foram avaliadas pelos instrumentos utilizados, temos que, nos 16 artigos acima citados, todos privilegiaram a mensuração de aspectos relacionados às "relações sociais"; todos menos um privilegiaram a dimensão "saúde física"; e as dimensões "estado psicológico" e "relações com as características do meio ambiente do indivíduo" foram, também, contempladas pela maioria dos artigos tendo sido citada em 13 artigos a primeira, e, em 11, a segunda. No entanto, no que se refere à dimensão "prontidão para o trabalho" e "nível de independência”, apenas cerca de $50 \%$ dos artigos se preocuparam com uma avaliação mais detida destes aspectos. Isto pode ser devido ao fato de que a média de idade da grande maioria dos indivíduos é superior a 55 anos e, assim sendo, deve-se evitar a confusão entre inabilidade para o trabalho ou para se tornar independente e fatores relacionados à terceira idade (tais como aposentadoria e doenças crônico-degenerativas comuns nessa população).

Quando a avaliação da qualidade de vida se deu sem a utilização de instrumentos, o que ocorreu em 54,3\% dos artigos, foi dada grande ênfase aos aspectos relacionados ao bem-estar físico, em detrimento de avaliações que contemplassem, também, o bem-estar social e psicológico. Apenas quatro destes 19 artigos contemplaram questões que avaliaram as relações sociais e nenhum deles se preocupou diretamente com a avaliação de aspectos psicológicos. 
Em consonância com as recomendações teóricas acerca do conceito "qualidade de vida" (Bowling, 1995a, 1995b; WHO, 1993), 77,14\% dos artigos estudados consideraram respostas emitidas pelos pacientes, o que se coaduna com a necessidade de se tratar qualidade de vida como um conceito permeado pela percepção pessoal do avaliado.

Merece destaque, por fim, o fato de que apenas $40 \%$ dos estudos sugeriram estratégias/condutas terapêuticas voltadas exclusivamente para a melhoria da qualidade de vida dos indivíduos estudados. Tal fato parece ter grande probabilidade de estar relacionado à imprecisão que a avaliação da qualidade de vida apresenta nos estudos arrolados.

Os achados da presente pesquisa parecem demonstrar que, em sua maioria, os estudos que se referem à avaliação da qualidade de vida em pacientes laringectomizados não o fazem com o rigor metodológico necessário para tanto. Na maior parte dos casos a dimensão saúde física é privilegiada em detrimento das outras dimensões imprescindíveis para a avaliação de tal constructo. Além disso, constatase que os estudos que pretendem obedecer aos preceitos metodológicos exigidos para tanto ainda o fazem, em sua maioria, de maneira incipiente. Afinal, foi constatado que a construção de instrumentos de domínios específicos se apresenta bastante aquém do pretendido por investigações que almejam consolidar os seus achados por meio de ferramentas de magnitude considerável (Batel-Copel et al., 1997; Boer et al., 1999). Além disso, deve-se considerar, também, o que assinalam Batel-Copel et al. (1997:31): " a despeito de um crescente interesse dos oncologistas na qualidade de vida, ela é ainda raramente incluída como um objetivo em seus ensaios clínicos".

Decerto que devem ser consideradas limitações também neste estudo, particularmente no que se refere à construção do QAQ que, apesar de ter alcançado validade de face aceitável para a continuidade da pesquisa, não teve robustez suficiente para que fosse excluída a possibilidade de decisões subjetivas. A confiabilidade interobservador do QAQ foi robusta o suficiente para que não houvesse maiores preocupações acerca deste aspecto. Entretanto, o tamanho de amostra, alcançado pelos artigos selecionados, não é grande o suficiente para gerar uma outra amostra para a avaliação da confiabilidade interobservador que dê conta deste parâmetro com maior precisão. Afinal, mesmo tendo sido avaliada sobre cerca de $23 \%$ do total de artigos (oito estudos selecionados aleatoriamente - por sorteio), a confiabilidade alcançada tem um intervalo de confiança amplo o suficiente para revelar sua fragilidade.

Sendo a qualidade de vida um constructo que comporta uma ampla variabilidade em sua definição, a avaliação do QAQ levou os avaliadores a apontar a necessidade de busca de informações não disponíveis para seu preenchimento e gerou dúvidas no que se refere à capacidade de o instrumento ser aplicado à diversidade de artigos sobre o tema e aferir um único domínio. Para amenizar este último inconveniente, o constructo "Qualidade de Vida" foi avaliado em todos os artigos, tendo como definição básica a que está declarada pelo WHOQOL.

No que se refere à busca bibliográfica, é fato, também, que, por mais abrangente e exaustiva que esta tenha sido, sempre ocorre uma perda de artigos que podem ter forte influência nos achados do estudo que se pretendeu realizar. Apesar de a busca manual não ter trazido acréscimos à amostra inicialmente selecionada, é importante frisar que a busca eletrônica pode ter sido prejudicada pela própria definição do termo qualidade de vida. Como este constructo, enquanto indexador, por exemplo, é sabidamente recente, é bastante provável que estudos que tratam de qualidade de vida por uma sinonímia não avaliada na busca eletrônica desta pesquisa tenham sido excluídos inintencionalmente deste trabalho.

\section{Conclusões e sugestões para pesquisas futuras}

Os achados do presente trabalho dirigem as expectativas acerca de pesquisas sobre qualidade de vida e pacientes laringectomizados para a necessidade de serem utilizados e construídos, dentro de padrões e rigores metodológicos, instrumentos apropriados para a avaliação deste conceito. É bem verdade que qualidade de vida pode ser um conceito bastante subjetivo, mas a subjetividade não pode ser impedimento para que sejam aprimoradas as técnicas de mensuração de tal constructo, visto que isto poderia conduzir à estagnação deste tipo de investigação.

Fazer constar dos artigos publicados a possibilidade de ocorrência de vieses e de variáveis de confusão se torna necessário, uma vez que tais características metodológicas são de fundamental importância para o reconhecimento da pertinência dos estudos realizados.

Por fim, a realização de estudos de coorte (prospectivos) pode ser de grande valor para a avaliação de tal constructo, na proporção em 
que qualidade de vida avaliada em estudos com outros tipos de desenho (p. ex.. estudos transversais) pode estar fortemente enviesada, seja por viés de sobrevida, na medida em que esses estudos acessam a população que foi laringectomizada e que sobreviveu, seja por viés de memória, porque um fato tão pregnante quanto a laringectomia pode estar influenciando as respostas emitidas pelos pacientes em relação a dados tão singulares quanto os que são investigados por este tipo de pesquisa.

\section{Referências}

ANELLO, C. \& FLEISS, J. L., 1995. Exploratory or analytic meta-analysis: Should we distinguish between them? Journal of Clinical Epidemiology, 48:109-116.

BATEL-COPEL, L. M.; KORNBLITH, A. B.; BATEL, P. C. \& HALLAND, J. C., 1997. Do oncologists have an increasing interest in the quality of life of their patients? A literature review of the last 15 years. European Journal of Cancer, 33:29-32.

BOER, M. F.; McCORMICK, L. K.; PRUYN, J. F. A.; RYCKMAN, R. M. \& BORNE, B. W., 1999. Physical and psychosocial correlates of head and neck cancer: A review of the literature. Otolaryngology Head and Neck Surgery, 120:427-436.

BOWLING, A., 1995. Measuring Disease. Buckingham: Open University Press.

CAMILLERI-BRENNAN, J. \& STEELE, R. J., 1998. Quality of life after treatment for rectal cancer. British Journal of Surgery, 85:1036-1043.

CATTARUZZA, M. S.; MAISONNEUVE, P. \& BOYLE, P., 1996. Epidemiology of laryngeal cancer. European Journal of Cancer. Part B, Oral Oncology, 32B:293-305.

DEVITA, V. T. J.; HELLMANN, S. \& ROSENBERG, S. A., 1997. Cancer: Principles \& Practice of Oncology. Philadelphia: Lippincott Williams \& Wilkins.

GOTAY, C. C. \& MURAOKA, M. Y., 1998. Quality of life in long-term survivors of adult-onset cancers. Journal of the National Cancer Institute, 90:656667.

GUNZBURGER, M., 1997. Psicoterapia: Redescobrindo a vida e a fala. In: Câncer de Laringe: Uma Abordagem Multidisciplinar (M. J. R. Noronha \& F. L. Dias, org.), pp. 323-326, Rio de Janeiro: Revinter.

JENICEK, M., 1989. Meta-analysis in medicine. Where we are and where we want to go. Journal of Clinical Epidemiology, 42:35-44.

\section{Agradecimentos}

As autoras agradecem à Mônica Campos, à Sandra Fonseca e aos especialistas anônimos pela participação no processo de avaliação da confiabilidade e da validade do instrumento da pesquisa.
KLIGERMAN, J.; SÁ, G. M.; FREITAS, R. Q. \& SOARES, J. R. N., 1992. Câncer de Laringe. Rio de Janeiro: Instituto Nacional do Câncer, Ministério da Saúde. LOPEZ-ABENTE, G.; POLLAN, M.; MONGE, V. \& MARTINEZ-VIDAL, A., 1992. Tobacco smoking, alcohol consumption, and laryngeal cancer in Madrid. Cancer Detection and Prevention, 16:265271.

MATHIESON, C. M.; STAM, H. J. \& SCOTT, J. P., 1990. Psychosocial adjustment after laryngectomy: A review of the literature. Journal of Otolaryngology, 19:331-336.

MOHIDE, E. A.; ARCHIBALD, S. D.; TEW, M.; YOUNG, J. E. \& HAINES, T., 1992. Postlaryngectomy quality-of-life dimensions identified by patients and health care professionals. American Journal of Surgery, 164:619-622.

MONTAZERI, A.; GILLIS, C. R. \& McEWEN, J., 1998. Quality of life in patients with lung cancer. Chest, 113:467-481.

MORENO, A. B., 1999. Avaliação da Qualidade de Vida em Pacientes Laringectomizados: Uma Revisão Sistemática. Dissertação de Mestrado, Rio de Janeiro: Instituto de Medicina Social, Universidade do Estado do Rio de Janeiro.

MUSCAT, J. E. \& WYNDER, E. L., 1992. Tobacco, alcohol, asbestos, and occupational risk factors for laryngeal cancer. Cancer, 69:2244-2251.

NORONHA, M. J. R. \& DIAS, F. L., 1997. Epidemiologia - Etiopatologia e fatores etiológicos do câncer da laringe - Potencial para quimioprevenção. In: Câncer da Laringe: Uma Abordagem Multidisciplinar (M. J. R. Noronha \& F. L. Dias, org.), pp. 47, Rio de Janeiro: Revinter.

OLIVEIRA, F. J., 1998. O Colesterol Total Sérico como Fator de Risco para o Desenvolvimento de Doença Cardíaca Coronariana em Idosos: Uma Meta-Análise Qualitativa. Dissertação de Mestrado, Rio de 
Janeiro: Instituto de Medicina Social, Universidade do Estado do Rio de Janeiro.

OXMAN, A. D. \& GUYATT, G. H., 1988. Guidelines for reading literature reviews. Canadian Medical Association Journal, 138:697-703.

OXMAN, A. D. \& GUYATT, G. H., 1991. Validation of an index of the quality of review articles. Journal of Clinical Epidemiology, 44:1271-1278.

OXMAN, A. D. \& GUYATT, G. H., 1993. The science of reviewing research. Annals of the New York Academy of Sciences, 703:125-134.

SACKS, H. S.; BERRIER, H.; REITMAN, D. \& CHALMERS, T. C., 1987. Meta-analyses of randomized controlled trials. New England Journal of Medicine, 316:450-455.

SPSS INCORPORATION, 1998. SPSS for Windows. Statistical Package for Social Sciences. Release 8.0. Chicago: SPSS Inc.

STREINER, D. L. \& NORMAN, G. R., 1989. Health measurement scales: A practical guide to their development and use. Oxford: Oxford University Press.

WHO (World Health Organization), 1993. WHOQOL Focus Group Work <http://www.who.int/mas/ $\mathrm{mnh} / \mathrm{mhp} / \mathrm{ql} . \mathrm{htm}>$.
WHO (World Health Organization), 1994. Epi Info Version 6.02. A Word Processing, Database, and Statistics System for Epidemiology on Microcomputers. Geneva: WHO.

WHO (World Health Organization)/IARC (International Agency for Research on Cancer), 1999. Cancer Mondial. Information on Cancer Incidence, Mortality and Survival. WHO Databank. <http:// www-dep.iarc.fr>.

ZATONSKI, W.; BECHER, H.; LISSOWSKA, J. \& WAHRENDORF, J., 1991. Tobacco, alcohol, and diet in the etiology of laryngeal cancer: A population-based case-control study. Cancer Causes and Control, 2:3-10.

ZHENG, W.; BLOT, W. J.; SHU, X. O.; GAO, Y. T.; JI, B. T.; ZIEGLER, R. G. \& FRAUMENI Jr., J. F., 1992. Diet and other risk factors for laryngeal cancer in Shanghai, China. American Journal of Epidemiology, 136:178-191.

Recebido em 28 de setembro de 2000

Versão final reapresentada em 3 de maio de 2001

Aprovado em 1 de agosto de 2001 